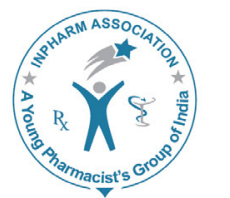

\title{
Evaluation of Adherence to Therapy in Patients of Type 2 Diabetes Mellitus
}

\author{
Smita Sontakke*, Mayur Jadhav, Sonali Pimpalkhute, Kavita Jaiswal and \\ Chaitali Bajait
}

Department of Pharmacology, Govt. Medical College, Nagpur, M.S. 440003, India.

\begin{abstract}
Objective: To evaluate adherence to therapy and study factors associated with non-adherence in patients of type 2 diabetes mellitus (DM). Methods: A prospective, cross-sectional, questionnaire based study was conducted in a tertiary care teaching hospital. 150 patients suffering from type 2 DM since six months or more were interviewed using self-designed, semistructured questionnaire to get information about adherence to medication, diet/exercise schedule and self-monitoring of blood glucose. Morisky medication adherence questionnaire was used to calculate overall adherence. Results: Average number of medicines taken by each patient daily was $4.75 \pm 1.73$ (mean $+\mathrm{SD}$ ). $70 \%$ patients reported non-adherence to medication schedule. Not buying all medicines $(58.66 \%)$, not taking prescribed dose of medicines $(34 \%)$, taking additional non-prescribed medicines (30\%) and not taking medicines for required duration $(25.33 \%)$ were common types of nonadherence. Unawareness about need of each medicine (55.66\%), forgetfulness $(50.66 \%)$ and high cost $(43.33 \%)$ were the common causes of non-adherence. In Morisky medication adherence questionnaire high, medium and low adherence was reported by $0 \%, 26 \%$ and $74 \%$ of patients, respectively. Moderately positive correlation was observed between poor adherence and number of concurrent illnesses, number of medicines taken and female gender. Regular monitoring of blood glucose and diet schedule was followed by $46 \%$ and $68 \%$ patients respectively. Conclusion: Since, not buying all medicines, which was mostly due to high cost was the commonest type of non-adherence, measures to provide free medicines to non-affording patients need to be implemented. Majority of patients were not aware about need of taking each medicine, hence, creating awareness about the same is essential for improving adherence to therapy.
\end{abstract}

Key words: Causes of medication non-adherence in DM, Medication non-adherence, Morisky medication adherence questionnaire, Polypharmacy in diabetes mellitus, Types of medication non-adherence in DM.

\section{INTRODUCTION}

Type 2 diabetes mellitus (DM) is a chronic disease that usually co-exists with other medical conditions. Uncontrolled DM leads to various short-term as well as

\begin{tabular}{|c|c|}
\hline \multicolumn{2}{|c|}{ Access this article online } \\
\hline Journal Sponsor & \multirow[b]{2}{*}{$\begin{array}{l}\text { Website: } \\
\text { www.jyoungpharm.org }\end{array}$} \\
\hline \multirow{2}{*}{ www.phcog net } & \\
\hline & $\begin{array}{l}\text { DOI: } \\
\text { 10.5530/jyp.2015.4s.8 }\end{array}$ \\
\hline
\end{tabular}

long term, microvascular and macrovascular complications. Hence, the patient may be taking multiple medications to treat several coexisting conditions. This creates a complex situation wherein the patient has to be on multiple drug therapy with complicated dosing schedules that is likely to result in low adherence to the medication plan. ${ }^{1}$ Adherence to the medication schedule is of utmost importance in successful management of chronic diseases. Adherence has been defined as the extent to which individuals follow the instructions they are given for prescribed treatments. ${ }^{2}$

\footnotetext{
*Address for correspondence:

Dr. Smita Sontakke, Associate Professor, Department of Pharmacology, GMC Nagpur, Address: 502, Silver Palms, Q-9 Laxminagar,

Nagpur.India.E-mail: smitaavanti@yahoo.co.in
} 


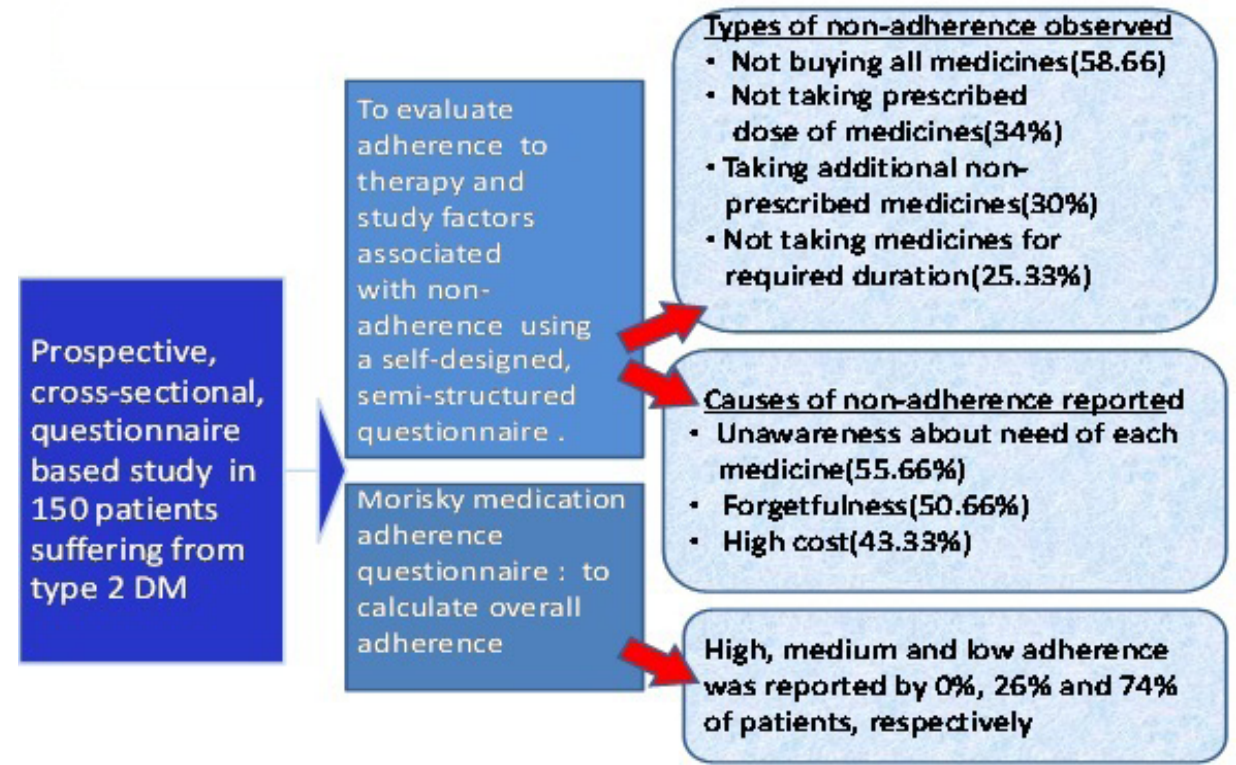

Graphical Abstract

As much as $30-60 \%$ of patients with chronic illnesses have been reported to be non adherent to drug therapy in various settings. ${ }^{3}$ According to World Health Organization estimates only half of people with chronic diseases are medication adherent. ${ }^{4}$ Widely variable rates of nonadherence are reported in various studies depending on the type of instrument used to measure adherence. Various methods are used to measure adherence, but none of these are suitable in all situations, each having its own pros and cons. Hence, no method is considered the gold standard. ${ }^{1}$

Non-adherence to medication is common in patients of type $2 \mathrm{DM}$ making glycemic control difficult to achieve. Inadequate glycemic control over a prolonged period increases the chances of disease related complications resulting in physical, mental and financial suffering to the patients. Highly variable rates of adherence to medication in patients of diabetes have been reported in different studies. Patient-reported adherence rates for medication of $83 \%$ and $78 \%$ in type 1 and type 2 diabetic patients respectively were reported in the Cross-National Diabetes Attitudes, Wishes, and Needs (DAWN) Study. ${ }^{5}$ In two studies in adult patients of type 2 diabetes mellitus $47 \%$ and $45.1 \%$ of patients were adherent with their study drug over the 1-year follow-up period. ${ }^{6,7}$

Studying only medication adherence in patients of diabetes would not be adequate as adherence to other components of the treatment regime such as blood glucose monitoring, diet and exercise schedule is also equally important as each of these components plays a vital role in the successful management of this chronic illness. Hence, it is important to assess the level of adherence to each component of the treatment regimen independently.

The cause of poor adherence is complex, multifaceted, and varies with each situation and patient. Hence, it is necessary to understand non-adherence to medication in a particular disease and study the factors affecting it. Various causes have been reported such as complex medication schedule, high cost of treatment, fear of adverse effects, lack of belief in the treatment, psychological problems etc. ${ }^{8}$ Variables such as age, medication knowledge and co morbidities were also shown to be associated with nonadherence. ${ }^{7}$ The nature of the clinical setting has also been reported to have an effect on patient adherence to treatment so it is also necessary to understand the problems specific to that setting. ${ }^{9}$

With this background, this study was planned to determine adherence to medication, blood glucose monitoring, dietary schedule and exercise and to study factors associated with non-adherence in patients of type $2 \mathrm{DM}$.

\section{MATERIAL AND METHODS}

This was a prospective, cross-sectional questionnaire based study initiated after approval of the institutional ethics committee. It was conducted in diabetic clinic of a tertiary care teaching hospital. Patients suffering from type $2 \mathrm{DM}$ since six months or more, of either gender, above 18 years of age were included. Patients meeting the inclusion criteria were briefed about the trial and informed consent was obtained from those willing to participate. 


\begin{tabular}{|c|c|c|}
\hline \multirow{2}{*}{ Questions } & \multicolumn{2}{|c|}{ No. of respondents(\%) } \\
\hline & Yes & No \\
\hline Aware of medicines taken & $95(63.33)$ & $55(33.66)$ \\
\hline Aware about dose and route of medicine & $92(61.33)$ & $58(38.66)$ \\
\hline Aware about frequency of administration & $89(59.33)$ & $61(40.66)$ \\
\hline $\begin{array}{l}\text { Aware about precautions to be taken } \\
\text { while taking medicines }\end{array}$ & $85(56.66)$ & $65(43.33)$ \\
\hline $\begin{array}{l}\text { Aware that not taking medication would } \\
\text { affect in any way }\end{array}$ & $82(54.67)$ & $68(45.33)$ \\
\hline $\begin{array}{l}\text { Stopped taking any medicines prescribed } \\
\text { by doctor }\end{array}$ & $31(20.66)$ & $119(79.33)$ \\
\hline $\begin{array}{c}\text { Stopped any medicine due to adverse } \\
\text { effects }\end{array}$ & $27(18)$ & $123(82)$ \\
\hline
\end{tabular}

Study participants were interviewed by a direct face-toface interview and the information was entered in a semistructured, open-ended questionnaire to get the following details: demographic characteristics, medication details, adherence to medication, diet control, exercise, blood glucose monitoring, awareness about disease, long-term complications, importance of adherence to medication and other measures.

The questionnaire was pre-tested in five patients and suitable modifications were done. In addition, adherence to therapy was also assessed by using the Morisky 8-item medication adherence questionnaire. In this, adherence is graded as high, medium and low and a higher score indicates low adherence. A total of 150 patients were interviewed. Patients were approached while they were waiting for doctor's consultation so that they didn't need to spend additional time for this purpose. The data obtained was compiled and analyzed.

\section{Statistical Analysis}

Values are expressed as counts and percentages. Spearman correlation test was used to find correlation between adherence and age, gender, number of medicines, number of coexisting illnesses. Graph pad prism software version 6.01 was used to analyze data.

\section{RESULTS}

One hundred and fifty patients participated in the study out of which 94 were men and 56 women. Mean age of respondents was $62.09 \pm 10.44$ (mean \pm SD) years. Average number of medicines taken by each patient per day was $4.75 \pm 1.73$ (mean $\pm \mathrm{SD}$ ). Thirty six patients had coexisting hypertension, 12 had ischemic heart disease (IHD), 72 patients had both IHD and hypertension while 30 patients did not have any concurrent illness. One hundred and five
$(70 \%)$ patients admitted that they did not strictly follow the medication schedule as prescribed by their doctor, which means they were non-adherent to medication schedule.

Table 1 shows that $33.66 \%$ patients were not aware about the medicines taken. About $38.66 \%$ patients were not aware of the dose and route of administration and $40.66 \%$ patients were not aware of the frequency of administration of medicines. Eighteen percent patients stopped taking any medicine due to some adverse effect.

Figure 1 shows that not buying all the medicines was the commonest type of non-adherence followed by not taking the prescribed dose of medicines, taking additional non-prescribed medicines and not taking medicines for required duration.

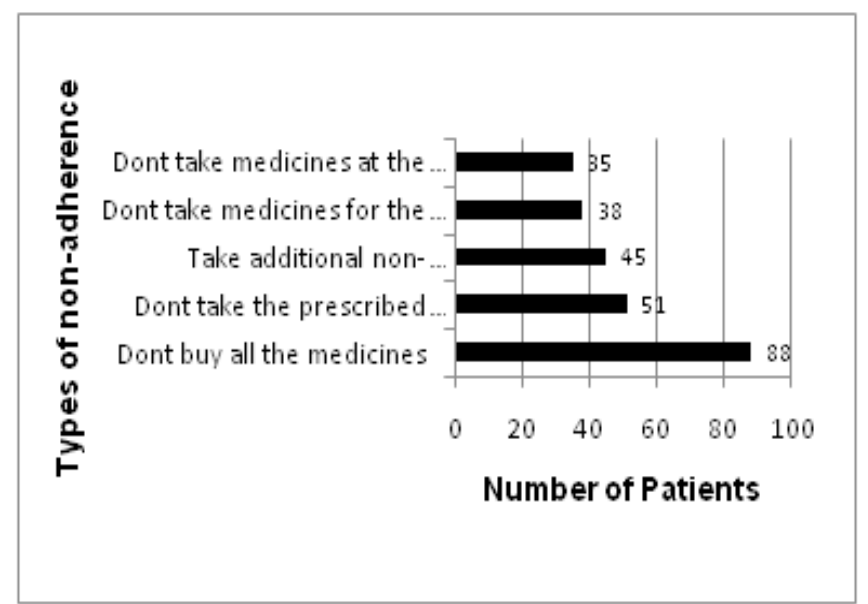

Figure 1: Types of non-adherence $(n=150)$

Figures in parentheses indicate percentage.

Figure 2 shows various reasons for non-adherence to medication in type 2 DM. It can be seen that unawareness about usefulness of each medicine taken, forgetfulness and high cost of medicines were the major reasons for non-adherence to medicines. 


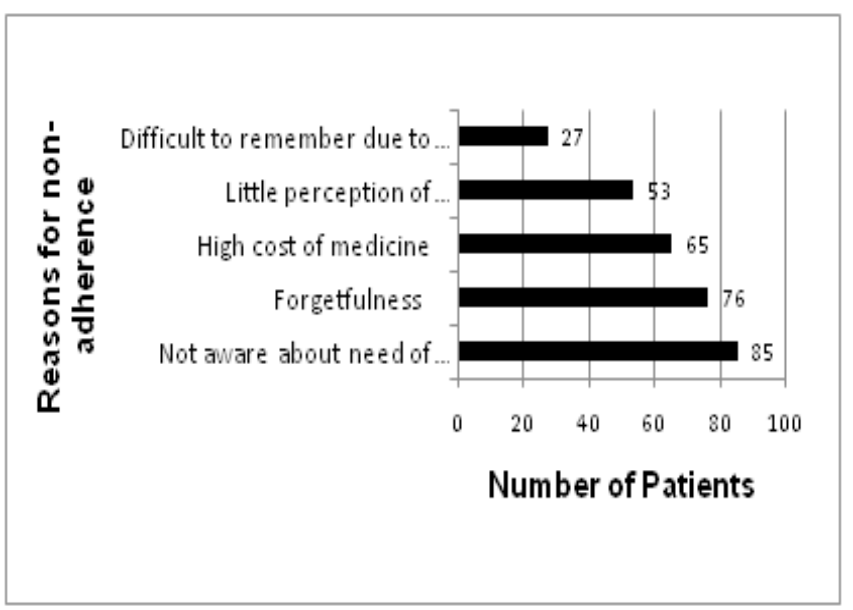

Figure 2: Reasons for non-adherence to medication $(n=150)$ Figures in parentheses indicate percentage.
Though $77.33 \%$ patients were aware about importance of blood glucose monitoring; only $46 \%$ patients actually monitored blood glucose regularly. Although $78 \%$ patients were aware about importance of diet control and exercise, but only $68 \%$ patients adhered to diet control and $49.33 \%$ patients followed exercise schedule Table 2.

Figure 3 shows level of adherence according to Morisky 8 -Item medication adherence questionnaire. It can be seen that none of the patients showed high adherence, 111 patients had low adherence, whereas 39 patients had medium adherence.

Forty two $\%$ patients were not aware of any consequences of non-adherence, $33 \%$ patients thought that non-adherence would lead to poor blood glucose control, whereas $25 \%$ patients thought that it would lead to worsening of disease.

\begin{tabular}{|ccc|}
$\begin{array}{c}\text { Table 2: Respondents' knowledge and practice about non medication factors }(\mathrm{n}=150) \\
\text { Questions }\end{array}$ & $\begin{array}{c}\text { No. of respondents }(\%) \\
\text { Yes }\end{array}$ & No \\
$\begin{array}{c}\text { Aware about importance of blood glucose } \\
\text { monitoring }\end{array}$ & $116(77.33)$ & $34(22.67)$ \\
\hline $\begin{array}{c}\text { Aware about importance of diet control } \\
\text { and exercise }\end{array}$ & $117(78)$ & $33(22)$ \\
\hline $\begin{array}{c}\text { Monitor blood glucose regularly } \\
\text { Follow diet control }\end{array}$ & $69(46)$ & $81(54)$ \\
\hline Follow exercise schedule & $102(68)$ & $48(32)$ \\
\hline Regular follow-up with doctor & $74(49.33)$ & $76(50.67)$ \\
\hline
\end{tabular}

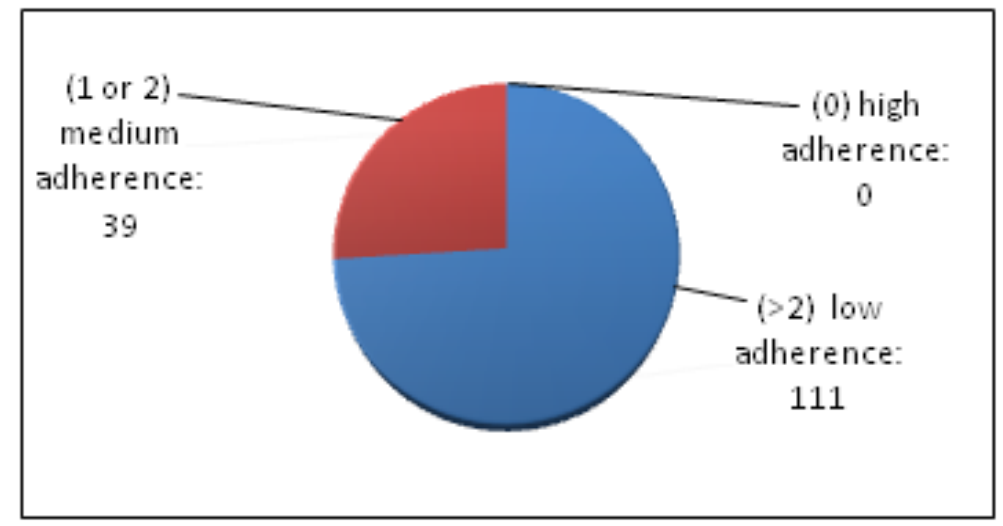

Figure 3: Level of adherence according to score on Morisky 8-Item Medication Adherence Questionnaire ( $n=150)$

Figures in parentheses indicate score on Morisky 8-Item Medication Adherence Questionnaire, figures indicate number of patients.

Moderately positive correlation was found between number of concurrent illnesses (Spearman r: 0.2499, $\mathrm{P}=0.002$ ), number of medicines taken (Spearman r: 0.6179, $\mathrm{P}<0.0001$ ) and adherence score (higher score denotes poor adherence). This indicates that adherence was inversely related to the number of concurrent illnesses and number of medicines taken. Higher adherence was also found to be moderately positively correlated to male gender (Spearman r: 0.2053, $\mathrm{P}=0.0117)$.

\section{DISCUSSION}

For good adherence to treatment patients must be able to follow the instructions they are given for prescribed 
treatments. It is important for the patients that they have adequate knowledge about the medicines they are prescribed, their dose, route and frequency. In this study it was found that $33.66 \%$ patients were not aware about the medicines taken. Patients should also be aware of various adverse effects of the medicines they are taking. This helps them to comply with the treatment. In this study 18\% patients stopped taking any medicine due to some adverse effect. Rwegerera GM also found $11.49 \%$ patients reporting adverse effects of medications as the reason for non-adherence. ${ }^{10}$

In this study, data obtained from the self-developed questionnaire showed that $70 \%$ patients were non-adherent to the prescribed medication schedule, while $74 \%$ patients showed low adherence in the Morisky medication adherence scale. Thus, nearly identical rates of non-adherence were obtained by the two methods. This may be because, though grading of adherence apparently appears to be different in these two methods; both methods are subjective, based on the patients' response. Widely variable rates of adherence to medication are reported in different studies. Two studies showed that adherence to oral medications in patients with type 2 diabetes was 53 and 67\% when measured by electronic monitoring. ${ }^{11,12}$ The DAWN study showed patient-reported adherence rates for medication in type 1 and type 2 diabetic patients of 83 and $78 \%$, respectively. ${ }^{5}$ In another study overall $45.1 \%$ of patients of type $2 \mathrm{DM}$ were adherent with their study drug over the 1-year follow-up period. In this study adherence was measured using data for prescriptions dispensed and since it was not known whether patients actually took the medications, hence the authors considered that adherence may have been overestimated. ${ }^{6}$ In another study adherence to medication was measured by using the Medication Compliance Questionnaire and approximately $53 \%$ of patients in the study population were found to be nonadherent. ${ }^{7}$

One of the major reasons for such variable rates of nonadherence reported in different studies is that there is no standard, universally accepted definition of adherence due to which patients may report adherence according to their own personal definitions rather than conventional medical concepts of non-adherence. ${ }^{13,14}$ Another reason maybe overestimation of adherence by patients which is difficult to study and presently poorly documented. Reasons for overestimation are obvious and may include difficulty recollecting the details of taking medicines, to satisfy the health care professionals, to avoid conflict or a combination of all of these.

The rates of adherence reported in our study are significantly low as compared to those reported earlier.
Various reasons quoted for non-adherence such as unawareness about the need of each medicine, high cost of medicines, forgetfulness, unawareness about the seriousness of the condition to be treated reflect patients' lack of knowledge and poor affordability. Health care professionals can play a major role in creating awareness amongst patients which can help to a great extent in improving adherence to medication. Complex dosing schedule was another reason for non-adherence. The acceptance of a medication regimen by a patient can be predicted, to a great extent, based on the complexity of regimen, cost, and the common adverse effects of that particular medication. ${ }^{1}$

In a one-year follow-up study evaluating 37,431 patients with type $2 \mathrm{DM}$ patients treated with monotherapeutic sulfonylurea or metformin had about $65 \%$ more adherent days (continuous and persistent use) than did patients treated with combination therapy. At the end of one year, only about $15 \%$ of the patients treated with monotherapy and $<5 \%$ of the patients treated with combination therapy took their medication continuously and persistently. This study further emphasizes the importance of simplification of regimen in the treatment of a chronic illness like DM. ${ }^{15}$

Common types of non-adherence reported were not buying all medicines, not taking the prescribed dose, not taking medicines at the prescribed time or for the prescribed duration. Apart from other reasons as mentioned earlier, cost of medicines is one of the major factors responsible for most of these. Whether high cost of medicines would be responsible for non-adherence would depend upon whether the patient pays the price out-of-pocket or it is covered by some form of medical insurance. Though this study was carried out in a government set-up, very often many medicines are not available in the hospital and patients have to purchase them from private pharmacies and since majority of the patients are not covered by any form of health insurance, they have to pay out-of-pocket for medicines. Hence, cost of medicines can be considered one of the major factors influencing adherence to medications in these patients. Prescribing low-cost medicines to such patients may help in improving adherence to a certain extent. ${ }^{1}$ In an earlier study $52.7 \%$ diabetic patients reported high cost of medications as the reason for non-adherence. ${ }^{10}$

Poor adherence was found to have a moderately positive correlation with number of concurrent illnesses and number of medicines prescribed. As is well known the treatment plan plays an important role in the likelihood of a patient adhering to treatment. Less complex treatment regimens with fewer medicines are likely to be associated with 
higher rates of adherence, as are fixed-dose combinations for those patients requiring combination therapy. ${ }^{9}$ In this study male gender was found to be associated with better adherence. Higher adherence to medication in male patients has also been reported earlier. ${ }^{6}$

In the evaluation of adherence to treatment in patients of diabetes, it is important to assess the level of adherence to each component of the treatment regimen independently, which includes: diet, physical activity, self-monitoring of blood glucose (SMBG), life-style changes; instead of using a single measure to assess adherence to the overall treatment. This is important because adherence to one regimen component may be unrelated to adherence in other regimen areas suggesting that adherence is not an unidimensional paradigm. ${ }^{16,17}$ In this study $68 \%$ patients admitted to adhering to the prescribed diet while $49.33 \%$ followed exercise schedule. Research has shown better adherence for medication use than for lifestyle change. ${ }^{18}$ In other studies, adherence rates of $65 \%$ were reported for diet but only $19 \%$ for exercise. ${ }^{17}$ In another study in type 2 diabetic patients adherence rates observed for diet and exercise were $37 \%$ and $35 \%$, respectively. ${ }^{5}$ In a study conducted in India, dietary prescriptions were followed regularly by only $37 \%$ of patients, while in a study in the United States about half (52\%) followed a meal plan and only $26 \%$ of respondents followed a physical activity plan. ${ }^{19,20}$ Thus, overall better adherence rates for diet than for exercise are reported. Strict adherence to diet and exercise schedule form an integral part of diabetes management. Emphasizing the importance of these upon the patients is the responsibility of health care professionals. In general majority of patients are aware about the importance of these factors in successful management of this disease, but in spite of that they find it difficult to strictly adhere to these measures as this requires great motivation and self-control. That might be the reason that many patients find it easy to adhere to medication schedule than to diet or exercise plan as is reported in some studies. ${ }^{18}$ In contrast, in this study better adherence was reported to diet and exercise than to medication. The reason for this finding is difficult to explain but probably it may be due to the various factors, as discussed earlier, which are responsible for non adherence to medication and which do not affect adherence to dietary schedule or exercise.

One measure of overall adherence to diabetes-management regimens is the assessment of patterns of SMBG. ${ }^{1}$ In this study $46 \%$ patients admitted to monitoring blood glucose regularly. Despite the improved technology, however, patients often do not adhere well to this aspect of the diabetes regimen. A study using a large national sample of patients with type 2 diabetes found that $24 \%$ of insulintreated patients, $65 \%$ of those on oral medications, and $80 \%$ of those treated by diet and exercise alone either never performed SMBG or did so less than once per month. ${ }^{21}$ In a study conducted to assess patterns of SMBG in northern California, United States, $67 \%$ of patients with type 2 diabetes reported not performing SMBG as frequently as recommended..$^{22}$ Similar findings were reported in a study conducted in India, in which only $23 \%$ of study participants reported performing SMBG. ${ }^{19}$ There may be various reasons for inadequate SMBG, such as unawareness about the importance of regular SMBG and cost of the monitoring strips which the patients might not be able to afford. Research has shown that increased SMBG is associated with improved glycemic control. ${ }^{23}$ This is possible as adjustments in pharmacotherapy can be done based on blood glucose levels so as to achieve adequate glycemic control. Hence, it is important that patients be made aware about the importance of SMBG and encouraged to follow it as recommended.

From the above discussion it is obvious that health care professionals can play a major role in improving adherence to therapy in patients of DM. Listening to the patients, understanding their problems and helping them to resolve them is important though it may be difficult in a busy clinical setting particularly in a government hospital. In spite of that spending just a few minutes with the patients can go a long way in overcoming many barriers to adherence to therapy in patients of DM which would help in improving long term outcome of the disease.

\section{CONCLUSION}

To conclude, poor patient adherence to treatment regimens is a major problem in the management of diabetes. Patients' lack of knowledge, poor affordability and forgetfulness are the major causes of non adherence. Adherence to therapy in DM needs a multidimensional approach which apart from medication includes adherence to prescribed diet and exercise schedule. Health professionals can play a major role in improving adherence to therapy by increasing interaction with patients.

\section{ACKNOWLEDGEMENT}

We thank Dr. Avinash Turankar, Associate Professor in Pharmacology Government Medical College Nagpur, India for helping in Statistical analysis. 


\section{ABBREVIATION}

DM: $\quad$ Diabetes Mellitus
DAWN: Diabetes Attitudes Wishes and

Needs Study

SMBG: $\quad$ Self-monitoring of blood glucose

\section{Highlights of Paper}

- This paper gives an idea about the extent of non-adherence to medication, self-monitoring of blood glucose and exercise schedule in patients of type 2 diabetes mellitus.

- Various factors responsible for non-adherence are also highlighted.

- These results will help in planning interventions aimed at improving adherence to therapy in these patients.

\section{Author Profile}
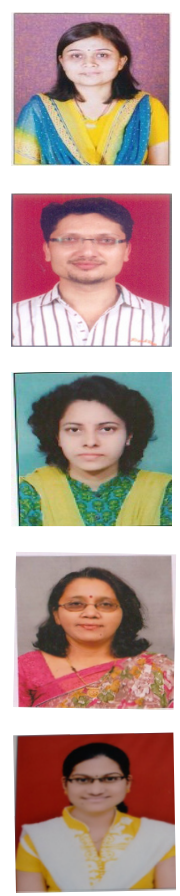

- Dr. Smita Sontakke: Is an Associate Professor in Pharmacology at GMC Nagpur, India. She has been working on various projects concerned with promoting rational use of medicines. She has conducted clinical trials on herbal medicines in diseases where modern medicines do not have much benefits, such as osteoarthritis. She is also involved in adverse drug reactions monitoring under the National Pharmacovigilance Programme.

- Dr. Mayur Jadhav: Has recently completed his MD in Pharmacology from GMC Nagpur, India. His topic of research during his post graduation was studying antiemetic effects of drugs in anticancer chemotherapy induced emesis.

- Dr. Sonali Pimpalkhute: Is an Assistant Professor in Pharmacology at GMC Nagpur, India. She is an co-opted member of Institutional Ethics Committee and actively involved in the functioning of Ethics Committee. She was granted funding for conducting clinical trials in HIV positive patients. She is also interested in working on quality of life studies in various chronic disorders.

- Dr. Kavita Jaiswal: Is an Assistant Professor in Pharmacology at GMC Nagpur, India. Apart from working on studies concerned with rational use of medicines she is also interested in working on psychopharmacological drugs.

- Dr. Chaitali Bajait: Is an Assistant Professor in Pharmacology at GMC Nagpur, India. She has conducted drug utilization studies in patients of chronic kidney disease. She has also worked on the controversial issue of prescribing zinc supplements to paediatric patients suffering from diarrhea.

\section{REFERENCES}

1. White JR. Improving Adherence in the Treatment of Type 2 Diabetes. US Pharm. 2010; 36(4): 11-5.

2. Clark M. Adherence to treatment in patients with type 2 diabetes. Journal of Diabetes Nursing 2004; 8(10): 386.

3. Rifkin DE, Barton Laws M, Madhumathi Rao VS, Balakrishnan Mark J, Sarnak, Ira Wilson B. Medication Adherence Behavior and Priorities Among Older Adults With CKD: A Semistructured Interview Study. Am J Kidney Dis. 2010; 56(3): 439-46.

4. World Health Organization: Adherence to long-term therapies: Evidence for action. Available at: http://www.who.int/chp/ knowledge/publications/adherence_report/en/. Last accessed on $17 / 03 / 2015$

5. Peyrot M, Rubin RR, Lauritzen T, Snoek FJ, Matthews DR, Skovlund SE. Psychosocial problems and barriers to improved diabetes management: results of the Cross-National Diabetes Attitudes, Wishes and Needs (DAWN) Study. Diabet Med. 2005; 22(10): 1379-85.

6. Curkendall SM, Thomas N, Bell KF, Juneau P, Weiss AJ. Predictors of Medication Adherence in Patients with Type 2 Diabetes Mellitus. Curr Med Res. Opin. 2013; 18(3 Suppl): S49-54.

7. Ahmad NS, Ramli A, Islahudin F, Paraidathathu T. Medication adherence in patients with type 2 diabetes mellitus treated at primary health clinics in Malaysia. Patient Prefer Adherence 2013; 17(7): 525-30.
8. Osterberg L, Blaschke T. Adherence to medication. N Engl J Med. 2005; 353(5): 487-97.

9. Nau DP. Recommendations for improving adherence to type 2 diabetes mellitus therapy--focus on optimizing oral and non-insulin therapies. Am J Manag Care 2012 ; 18(3 Suppl): S49-54.

10. Rwegerera GM. Adherence to anti-diabetic drugs among patients with Type 2 diabetes mellitus at Muhimbili National Hospital, Dar es Salaam, Tanzania-A cross-sectional study. Pan African Medical Journal 2014; 17: 252.

11. Mason BJ, Matsuyama JR, Jue SG. Assessment of Sulfonylurea adherence and metabolic control. Diabetes Educ.1995; 21(1): 52-7.

12. Paes AH, Bakker A, Soe-Angie CJ. Impact of dosage frequency on patient compliance. Diabetes Care 1997; 20(10): 1512-7.

13. Garber MA, Nau DP, Erickson SR, Aikens JE, Lawrence JB. The concordance of self-report with other measures of medication adherence: a summary of the literature. Medical Care 2004; 42(7): 649-52.

14. Rudd P. In search of the gold standard for compliance measurement. Arch Intern Med. 1979; 139(6): 627-8.

15. Dailey G, Kim MS, Lian JF. Patient compliance and persistence with antihyperglycemic drug regimens: evaluation of a medicaid patient population with type 2 diabetes mellitus. Clinical therapeutics $2001 ; 23(8): 1311-20$. 
16. Kurtz SMS. Adherence to diabetes regimens: empirical status and clinical applications. Diabetes Educ. 1990; 16(1): 50-6.

17. Kravitz RL, Hays RD, Sherbourne CD, DiMatteo MR, Rogers $\mathrm{WH}$, Ordway L, et al. Recall of recommendations and adherence to advice among patients with chronic medical conditions. Arch Intern Med. 1993; 153(16): 1869-78.

18. Anderson RM, Fitzgerald JT, Oh MS. The relationship of diabetesrelated attitudes and patients' self-reported adherence. Diabetes Educ. 1993; 19(4): 287-92.

19. Shobhana R, Begum R, Snehalatha C, Vijay V, Ramachandran A. Patients' adherence to diabetes treatment. J Assoc Physicians India 1999; 47(12): 1173-5.

20. Shultz JA, Sprague MA, Branen LJ, Lambeth S. A comparison of views of individuals with type 2 diabetes mellitus and diabetes educators about barriers to diet and exercise. J Health Commun. 2001; 6(2): 99-115.

21. Harris MI. Frequency of blood glucose monitoring in relation to glycemic control in patients with type 2 diabetes. Diabetes Care 2001; 24(6): 979-82.

22. Karter AJ, Ferrara A, Darbinian JA, Ackerson LM, Selby JV. Selfmonitoring of blood glucose: language and financial barriers in a managed care population with diabetes. Diabetes care 2000; 23(4): 477-83.

23. Strowig SM, Raskin P. Improved glycemic control in intensively treated type 1 diabetic patients using blood glucose meters with storage capability and computer-assisted analysis. Diabetes Care1998; 21(10): 1694-8. 